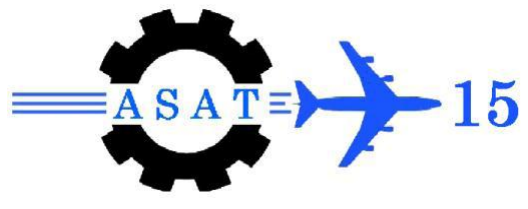

\title{
Multiple Antenna Selection of Multi-Antenna Relays Using MSC Scheme
}

\author{
Ahmed El-Mahdy ${ }^{*}$ and Ahmed Ali ${ }^{\dagger}$
}

\begin{abstract}
In this paper we analyze the performance of multiple relay networks when multiple antennas are deployed only at the relays. We apply minimum selection combining (MSC) scheme to select and combine the received signals at the relays. The channel is assumed to be Rayleigh flat fading. The performance of the network is investigated for known and estimated channel and for both decode-and-forward and amplify-and forward protocols. Channel estimation is performed using least square algorithm. The performance is compared with the performance of the network when the multiple antennas of the relays are combined using maximal ratio combiner (MRC). We show that reduction in complexity is achieved for decode-and-forward and amplify-and forward relaying protocols, with some sacrifices in performance.
\end{abstract}

Keywords: Wireless communication, cooperative diversity, and fading channel.

\section{Introduction}

Recently, there has been a growing interest in relay transmission for wireless systems. The basic idea is to communicate with the destination through the help of one or several intermediate terminals [1]. The major benefits of relay transmission include power saving, coverage extension, and reduction of interference levels. As a result, the users can enjoy better link quality and as such, improved quality of service, especially when the direct sourcedestination link is of poor quality. For multiple-antenna relay channels where every terminal in the network can be deployed with multiple antennas, studies are mainly concentrated on spatial multiplexing systems References [2]. Diversity combining techniques improve the performance of wireless communication system at the cost of increased power consumption; also diversity techniques improve the performance of wireless communication systems over fading channels by means of multiple transmission and/or reception of the same signal [3]. The optimal diversity-combining scheme is the well-known maximum ratio combining (MRC). Through coherent summation of properly weighted fading replicas of the same signal, MRC offers the largest possible performance improvement and the best bit error rate performance. This performance gain, however, comes at the cost of increased receiver hardware complexity and power consumption since the receiver needs to process all these replicas coherently. Over the past few years, there has been considerable interest in reduced complexity combining schemes. Generalized selection combining (GSC), also known as hybrid selection/maximum ratio combining (H-S/MRC), is one of the most widely studied schemes (see for example [4]-[5] and references therein). With GSC, the optimal MRC scheme is applied to a fixed number of the best paths of all available ones. Since not all diversity paths need to be processed in the MRC fashion, the receiver hardware complexity is

Professor in the faculty of information Engineering \& Technology (IET), German University in Cairo. ahmed.elmahdy@guc.edu.eg

$\dagger \quad 4^{\text {th }}$ year student in the faculty of IET; ahmed.El-nemer@student.guc.edu.eg . 
lower than traditional MRC scheme. In the meantime, the receiver with GSC still needs to keep all MRC branches always active during the data reception stage, which leads to a considerable amount of processing power consumption. While this extra power consumption may not be an issue for the base station, it certainly reduces the valuable battery time of the mobile devices. So the MS-GSC is used which is an intermediate between the MRC and the GSC, which has better complexity than the MRC but with less performance. The first theoretical study of MS-GSC scheme was carried out in [6], where the distribution of the number of combined paths N with MS-GSC was derived and the error performance of MSGSC was investigated. In [7], MS-GSC shows a low standard deviation in bit error statistics and advantages practical implementation. In [8] the performance of the MS-GSC scheme is analyzed over fading channels.

In this paper, the performance of the multi-antennas multi-relay network is investigated when the MS-GSC scheme is used to combine the received signals in each relay. The performance is investigated for the two famous protocols of the relay network; namely decode and forward and amplify and forward protocols. Moreover, the performance of the network is compared with the performance when the MRC is used for combining. The fading channels between the source and the relays and between the relays and the destination are estimated using least square algorithm. The performance of the channel estimation algorithm is evaluated in terms of the mean square error of estimation.

The rest of this paper is arranged as follows. Section 2 presents the system model under consideration. MSC scheme is presented in Section 3. The multi-antenna relays with MS-GSC is analyzed in Section 4. Computer simulation and the obtained results are presented in Section 5 and finally, conclusions are drawn in Section 6.

\section{System Model}

We consider a two-hop network model with one source, one destination and $K$ relays as shown in Fig. 1. We ignore the direct link between the source and the destination. It is also assumed that the source and the destination are deployed with single antennas while relay $\mathrm{k}$ is deployed with $m_{k}$ antennas; the total number of antennas at all relays is fixed to $\mathrm{N}$. This can be expressed as:

$$
\sum_{k=1}^{K} m_{k}=N
$$

The two famous protocols for the relay operation are considered; namely decode and forward and amplify and forward protocols. In decode and forward protocol, the source transmit the signal to the relays and each relay decodes the entire received message, re-encodes it and sends it to the destination. In this protocol, each relay has the same codebook as the source in order to be able to decode and re-encode the received message. In amplify and forward (AF), during the first interval, the transmitter sends the signal to the relays. Then each relay multiplies its received signal by a gain and during the second interval forwards the amplified signal to the destination. The drawback of this method is that, the noise at each relay is also amplified. This method is often used when the relays have limited resources, e.g. processing time or power, available.

We restrict our discussion to the case where the channels are frequency flat fading. The data transmission is over two time slots using two-hops. In the first transmission time slot, the source broadcasts the signal to all the relay terminals. The input/output relation for the source to the $k_{t h}$ relay is given by: 


$$
\mathbf{R}_{k}=\sqrt{E_{b}} \mathbf{h}_{k}^{s r} \mathbf{s}_{k}^{s r}+\mathbf{W}_{k}^{s r}
$$

where $\mathbf{R}_{\mathbf{k}}$ is the $m_{k} \square n$ receive signal matrix, where $\mathrm{n}$ denotes to the number of bits of the signal, $\mathbf{s}^{s r}$ is $\left(1^{\wedge} \mathrm{n}\right)$ vector represents the transmitted signal from the source to the relays which has unit mean power and $E_{b}$ denotes the transmit power at the source. In (2), $\mathbf{W}_{k}^{s r}$ is the $m_{k}$ $n$ complex circular additive white Gaussian noise (AWGN) vector at relay $\mathrm{k}$. The vector $\mathbf{h}_{k}{ }^{s r}$ is the $m_{k} \square$ l channel transfer vector from the source to the $k_{t h}$ relay.

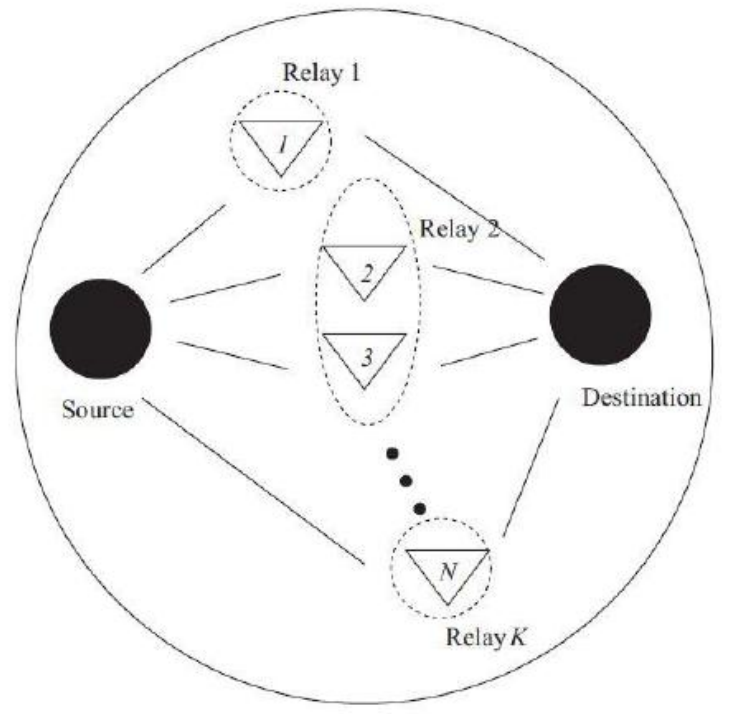

Fig.1 System model for a two-hop network: source and destination are each deployed with one antenna. A total of $\mathrm{N}$ antennas are deployed at $\mathrm{K}$ relays.

The entries of $\mathbf{h}_{k}{ }^{s r}$ are independent and identically distributed random variables. In the second hop, each relay processes its received signals after combining and re-transmits them to the destination. The signal received at the destination can be written as:

$$
\mathbf{Y}=\sum_{k=1}^{K} \mathbf{h}_{k}^{r d} \mathbf{s}_{k}^{r d}+\mathbf{W}_{k}^{r d}
$$

where $\mathbf{h}_{k}^{r d}$ is $m_{k} \times 1$ channel vector from the $k^{\text {th }}$ relay to the destination, each entry in this vector is an independent and identically distributed random variable and $\mathbf{W}_{k}{ }^{\text {rd }}$ is the complex additive white Gaussian noise at the destination with variance $N_{0} / 2$. The $1 n$ vector $\mathbf{s}_{k}^{r d}$ is the transmit signal vector at relay $\mathrm{k}$.

\section{Minimum Selection Combining Scheme}

MS-GSC system is arranging the received branches in descending order due to their signal to noise ratio, compare the 1 st received branch with the threshold if the signal to noise ratio of the received branch exceed the threshold then this will be the output of the system and it is the only case that system is working as selection combining (SC) system, if received branch did not pass the threshold then the system will combine the next branch so the output will be the first and the second branch combined then stop the process if the output (combined SNR) exceeds the threshold value. These processes save power consumption due to that not all received branch are activated. In Fig. 2, the mode of operation of MS-GSC receiver is described, where the receiver first estimates all received branches. Since all branches are arranged in descending order then the receiver start with the first branch knowing it's the branch with the best SNR Y1, the receiver tries to increase the combined SNR Yc above the threshold YT by increasing the number of the received branches. If Y1 > YT, then the only the strongest received branch will be the output of the system and If Y1 <YT, then the receiver will activate the next branch and combine them together. The output SNR is now Y1+ Y2 if it 
is above threshold, then only these two branches will be used as a output of the system, if it is less than the threshold the process continue and activate another one, this process is going till the combined SNR is above the threshold, or all received branches are combined. The previous process show that the MS-GSC system saves power consumption over other systems, as the system does not need to activate all the received branches in one time, in addition to not combining many weak branches that may consume power with no benefit, it also provide less complexity than MRC system.

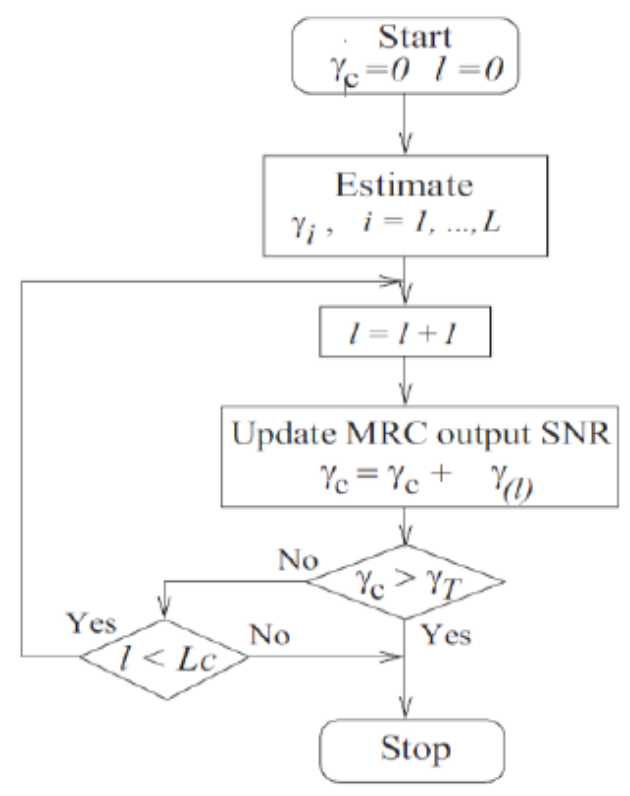

Fig. 2 Flow chart of MS-GSC mode of operation

The mode of operation of MS-GSC can be mathematically summarized as [10, Eq. 3]:

$$
\begin{aligned}
\gamma_{c}=\gamma_{(1)} \text { iff } \gamma_{(1)} \geq \gamma_{T} \\
\gamma_{c}=\gamma_{(1)}+\gamma_{(2)} i f f \gamma_{(1)}<\gamma_{T} \& \gamma_{(1)}+\gamma_{(2)} \geq \gamma_{T} ; \\
\quad \bullet \\
\bullet \\
\gamma_{c}=\sum_{j=1}^{i} \gamma_{(j)} \text { iff } \sum_{j=1}^{i} \gamma_{(j)}<\gamma_{T} \& \sum_{j=1}^{i} \gamma_{(j)} \geq \gamma_{T} ; \\
\cdot \bullet \\
\cdot \\
\gamma_{c}=\sum_{j=1}^{I_{c}} \gamma_{(j)} \text { iff } \sum_{j=1}^{I_{c}-1} \gamma_{(j)}<\gamma_{T}
\end{aligned}
$$

The average number of combined paths with MS-GSC can be given by [9]:

$$
\bar{N}=\sum_{i=1}^{I_{\varepsilon}} i \operatorname{Pr}[N=i]=1+\sum_{i=1}^{I_{c}-1} P_{\Gamma_{i}}\left(\gamma_{T}\right)
$$

where $\mathrm{N}$ is the average number of active hranches of the relay, $\gamma_{T}$ is the threshold, $L_{c}$ is the number of antennas in each relay and $P_{\mathrm{I}_{i}}()=.P_{\sum_{j-1^{\prime}}^{i} \gamma(j)}($.$) .$ 


\section{Analysis of Multi-Antenna Relays with MS-GSC}

In this section we apply MSC and MRC technique to the system model described in section II. We discuss both decode-and-forward and amplify-and-forward relaying modes below.

\section{A. Amplify-and-forward relaying}

We assume that each relay receiver perform MSC of the signal vector, it amplifies the signal by a factor that can meet the power constraint. The amplifying factor can be computed as [10]:

$$
G_{k}=\frac{E \frac{m_{k}}{N}}{E \sum_{i=1}^{N c}\left|h_{i, k}^{s r}\right|^{2}+N_{0}}
$$

where the summation from $i$ to $N_{c}$ is a random variable represents the number of the selected branches from the MSC combining scheme used at the relay (see (2)), and $N_{o}$ is the power spectral density. Unlike decode-and-forward protocol, the transmitted signal at the output of each relay is multiplied by the gain $G_{k}$. The signal to noise ratio at the output of the relay and at the destination can be written as:

$$
\gamma_{k}^{m_{k}}=\sum_{i=1}^{N_{c}}\left|h_{i, k}^{s r}\right|^{2}
$$

The received signal at $i_{t h}$ antenna of the $k_{t h}$ relay is given by:

$$
\mathbf{r}_{i, k}^{s r}=\sqrt{E_{b}} h_{i, k}^{s r} \mathbf{s}_{k}^{s r}+\mathbf{w}_{i, k}^{s r}, \quad i=1, \ldots . m_{k}
$$

Then the $(1 \square \mathrm{n})$ received vector at the relay $k$ after performing the MSC scheme is given by:

$$
\mathbf{r}_{k}^{s r}=\sum_{i=1}^{N_{c}} \mathbf{r}_{i, k}=\sum_{i=1}^{N_{c}}\left(\sqrt{E_{b}} h_{i, k}^{s r} \mathbf{s}_{k}^{s r}+\mathbf{w}_{i, k}^{s r}\right)
$$

where $h_{i, k}^{s r}$ denotes the fading channel parameter of the $i_{t h}$ antenna at relay $\mathrm{k}$, and $\mathbf{w}_{i, k}{ }^{s r}$ denotes the $(1 \times \mathrm{n})$ noise vector of the $i_{t h}$ branch and the $k_{t h}$ relay and $N_{c}$ is a random variable represents the number of selected and combined branches using MSC scheme. For MRC scheme, $N_{c}$ is equal to the total number of all branches at the $k_{t h}$ relay. The received signal at the destination from the $k_{t h}$ relay can be written as:

$$
\widetilde{\mathbf{R}}_{k}^{r d}=G_{k} \mathbf{h}_{k}^{r d} \mathbf{r}_{k}^{s r}+\mathbf{W}_{k}^{r d}
$$

where $\tilde{\mathbf{R}}_{k}^{r d}$ is the received matrix from relay $k$ at the destination before MS-GSC combining. The $\mathbf{h}_{k}{ }^{r d}$ is $m_{k} \square$ l channel transfer vector from relay to destination and $\mathbf{W}_{k}{ }^{r d}$ is $m_{k} \times n$ which is the additive noise in channel from the relay to destination. The destination receiver receives the sum of the signals from all K relays and performs data detection. Using (6) and (10), the output signal at the destination can be written as:

$$
\widetilde{\mathbf{r}}=\sum_{k=1}^{K} \sum_{i=1}^{N c}\left(\frac{E \frac{m_{k}}{N}}{E \sum_{i=1}^{N c}\left|h_{i, k}^{s r}\right|^{2}+N_{0}} h_{i, k}^{r d} \mathbf{r}_{k}^{s r}+\mathbf{W}_{i, k}^{r d}\right)
$$

\section{B. Decode-and-forward relaying}

The received signal at the $i_{t h}$ antenna of the $k_{t h}$ relay is given by (8). After performing the MSC scheme, the received signal is given by (9), then the received signals are decoded at the relays and then encoded again with BPSK modulation and then transmitted. The received signal at the destination from the $k_{t h}$ relay can be written as:

$$
\widetilde{\mathbf{Z}}_{k}^{r d}=\sqrt{E_{b}} \mathbf{h}_{k}^{r d} \hat{\mathbf{s}}+\mathbf{W}_{k}^{r d}
$$


The destination receiver receives the sum of the signals from all $\mathrm{K}$ relays and performs data detection. The output signal at the destination can be written as:

$$
\widetilde{\mathbf{z}}=\sum_{k=1}^{K} \sum_{i=1}^{N_{c}}\left(\sqrt{E_{b}} \mathbf{h}_{i, k}^{r d} \hat{\mathbf{s}}+\mathbf{W}_{i, k}^{r d}\right)
$$

\section{Channel estimation algorithm}

There are many methods that can be used to estimate the fading channel parameter; one of these methods is the Least Square Estimation algorithm (LSE). This algorithm is performed by sending a pilot signal $S_{Q}(\mathrm{t})$ known to the receiver and then the receiver apply the equation:

$$
\tilde{h}_{i}=\left(\boldsymbol{S}_{Q}^{T} \boldsymbol{S}_{Q}\right)^{-1} \boldsymbol{S}_{Q}^{T} \boldsymbol{r}_{i}
$$

where, $\boldsymbol{S}_{\boldsymbol{Q}}$ is the pilot signal vector containing the pilot sequence of bits and $\boldsymbol{r}_{\boldsymbol{i}}$ is the received vector that contains the pilot signal contaminated by noise and fading channel. The performance of the least square algorithm is measured in terms of mean square error (MSE) which is one of many ways used to measure the accuracy of the estimation algorithm. Mean square error defines the significance of the channel estimator and it is given by

$$
M S E=E\left(\left|\tilde{h}_{i}-h_{i}\right|^{2}\right)
$$

where $\mathrm{E}($.$) denotes the expectation.$

\section{Simulation and Results}

In this section, the performance of the multi-antenna multi-relays is evaluated when MS-GSC is used for selection and combining of received signals at the relays and at the destination. The simulation parameters are as follows. The number of bits is 10,000 , the number of antennas is four in both sides of the relay, the type of modulation is BPSK, and the channel is flat fading channel. The value of the threshold of MSC is 0.01 . The performance of the network is evaluated for both amplify and forward and decode and forward protocols and for known and estimated channel.

The performance comparison of decode and forward protocol using MSC and MRC is shown in Figs. 3 and 4 for known and estimated channel respectively. The results show that there is a gap in BER performance of decode and forward protocol using MSC and MRC. This is of course usual, because the performance using MRC is considered as a lower bound of performance of all diversity combining techniques. But this optimum performance comes at the expense of complexity. In our case, the gap in performance is not exceeding $3 \mathrm{~dB}$ at $\mathrm{BER}=10^{-4}$. We can sacrifice with the $3 \mathrm{~dB}$ to achieve a good power saving and a great reduction in complexity. By comparing the results of Fig. 3 and Fig. 4, we note that the performance of decode and forward protocol with known channel is better than the performance with estimated channel. This is because the channel estimation errors affect the BER performance.

Similar conclusions can be drawn from Fig. 5 which shows the performance comparison of amplify and forward protocol using MSC and MRC for estimated channel.

The figure shows that the performance of the MSC and MRC curves are better than the curves obtained at Fig. 3 due to that the signal is decoded again at the relay and no gain is multiplied by the signal so the noise did not amplified. 


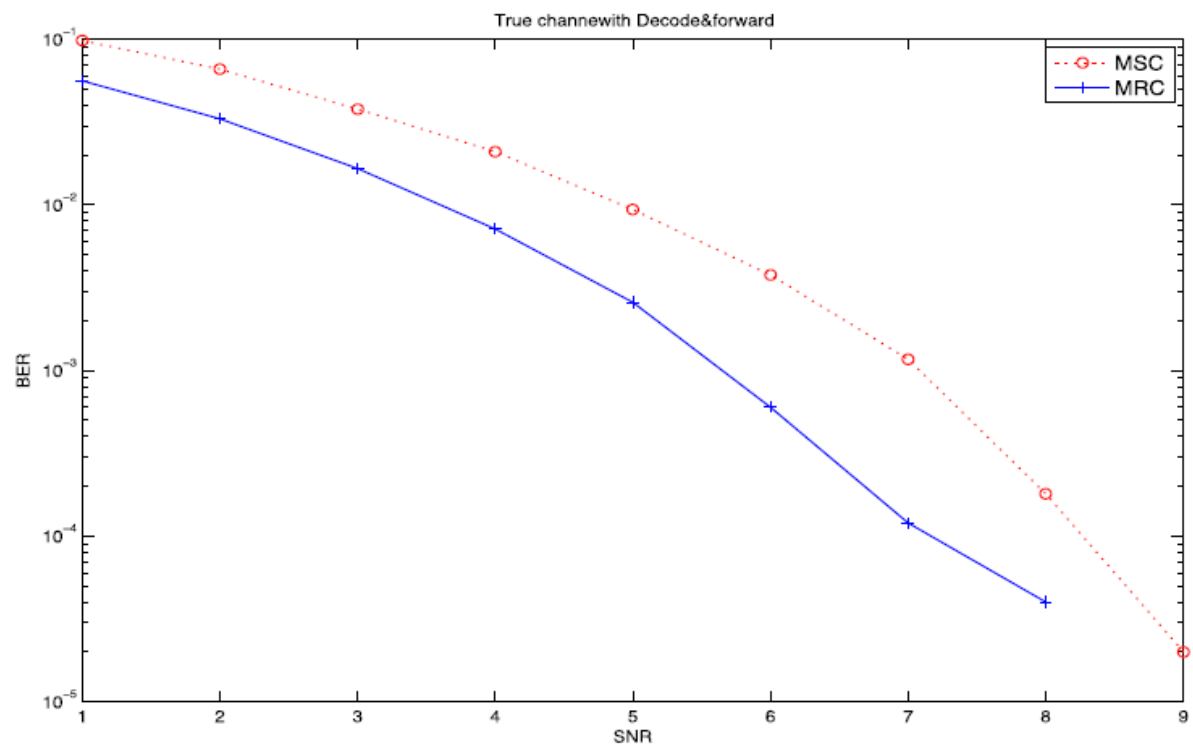

Fig. 3 Performance comparison of decode and forward protocol using MSC and MRC for known channel

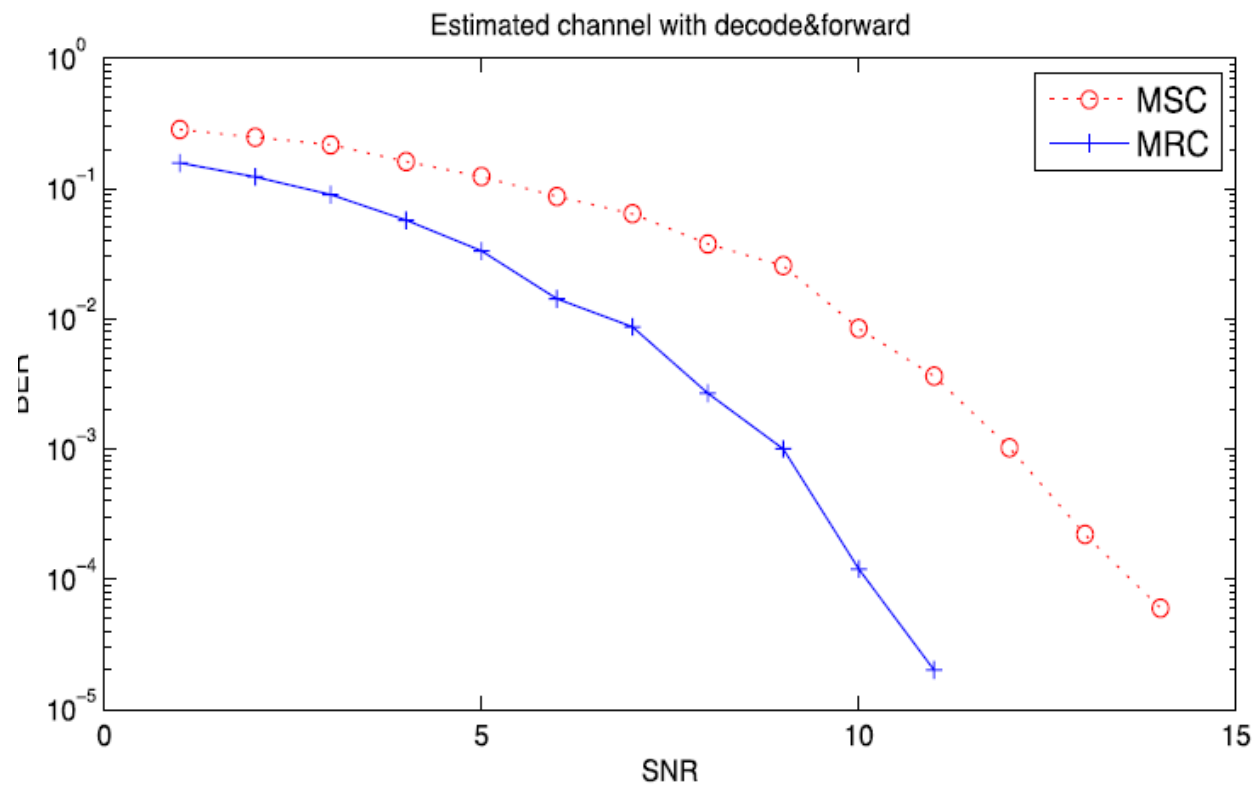

Fig. 4 Performance comparison of decode and forward protocol using MSC and MRC for estimated channel

The LS algorithm is used to estimate the channels between the source and every antenna in the relays and between every antenna of the relays and destination. We provide only one channel estimate as an example and the rest of the channels are the same. Figure 6 illustrates the absolute of perfect and estimated channel from source to first antenna of one relay. The number of pilot symbols used to produce this figure is 100 bits. The results show that at low SNR, the noise dominates the performance and there is a gap between the true and estimated channel. When SNR increases, the estimated channel converges to the true one. 


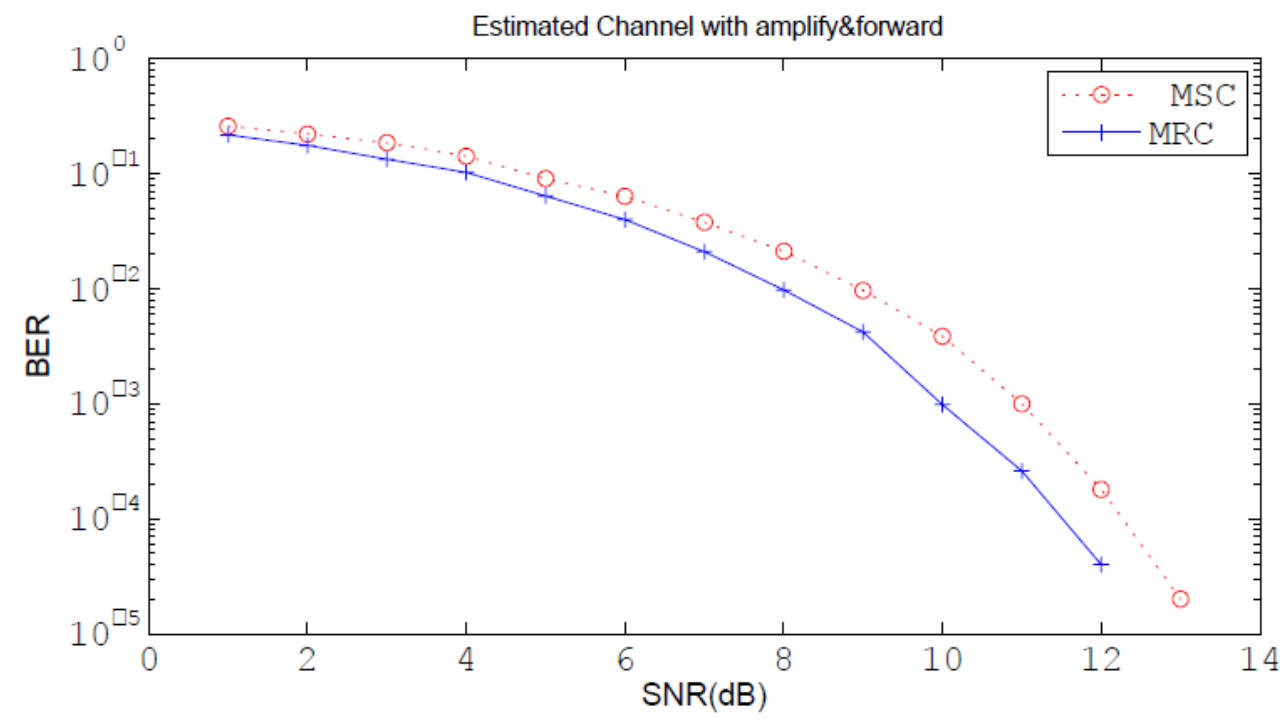

Fig. 5 Performance comparison of amplify and forward protocol using MSC and MRC for estimated channel

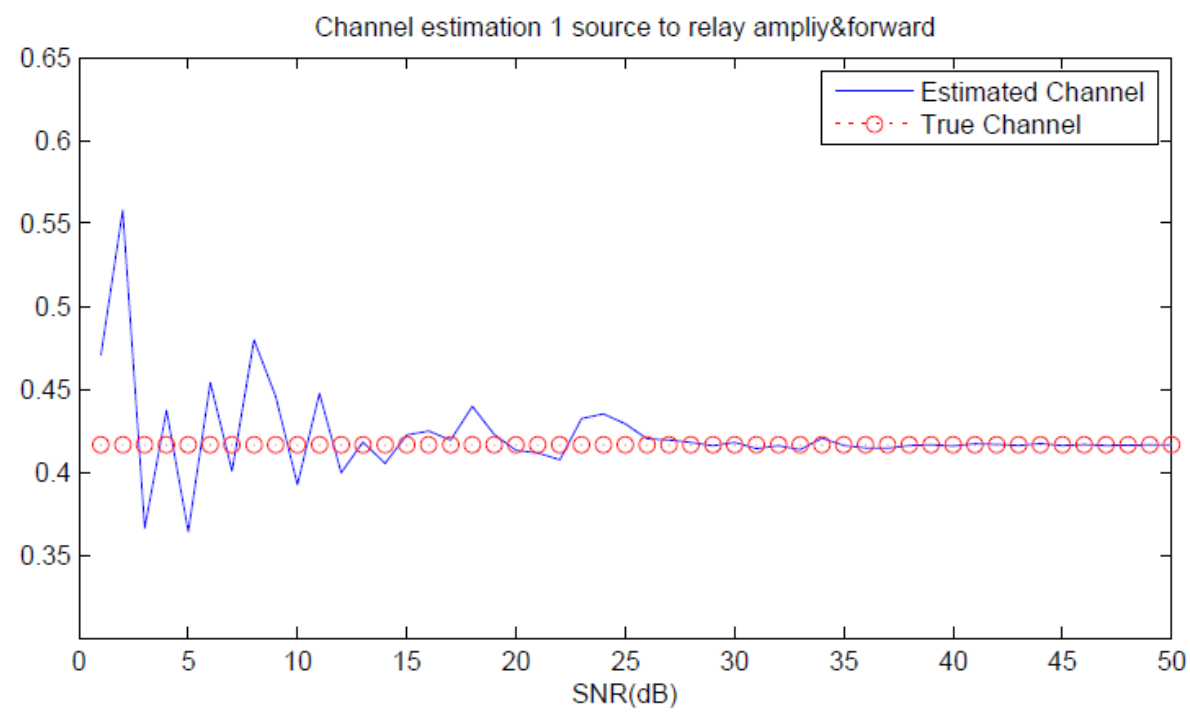

Fig. 6 Absolute values of true and estimated channel from source to first antenna of one relay

\section{Conclusions}

In this paper, the performance of multi-antenna multi-relays network is investigated when the MSC scheme is used to select and combine a number of antennas of the relays. Channel estimation is performed using least square algorithm. The results show that a reduction in complexity and power consumption can be achieved when combining the best branches using MSC scheme. The loss in performance is about $3 \mathrm{~dB}$ from the MRC which combines all the branches.

Another measure of channel estimation accuracy is the normalized mean square error of estimation which is plotted in Figs. 7. This figure is obtained by calculating the square error for 4 channels between relays and destination, and then mean of them is evaluated to obtain NMSE of estimation. The results show that the NMSE of estimation decreases as SNR increases until reach small values after $S N R=15 \mathrm{~dB}$. 


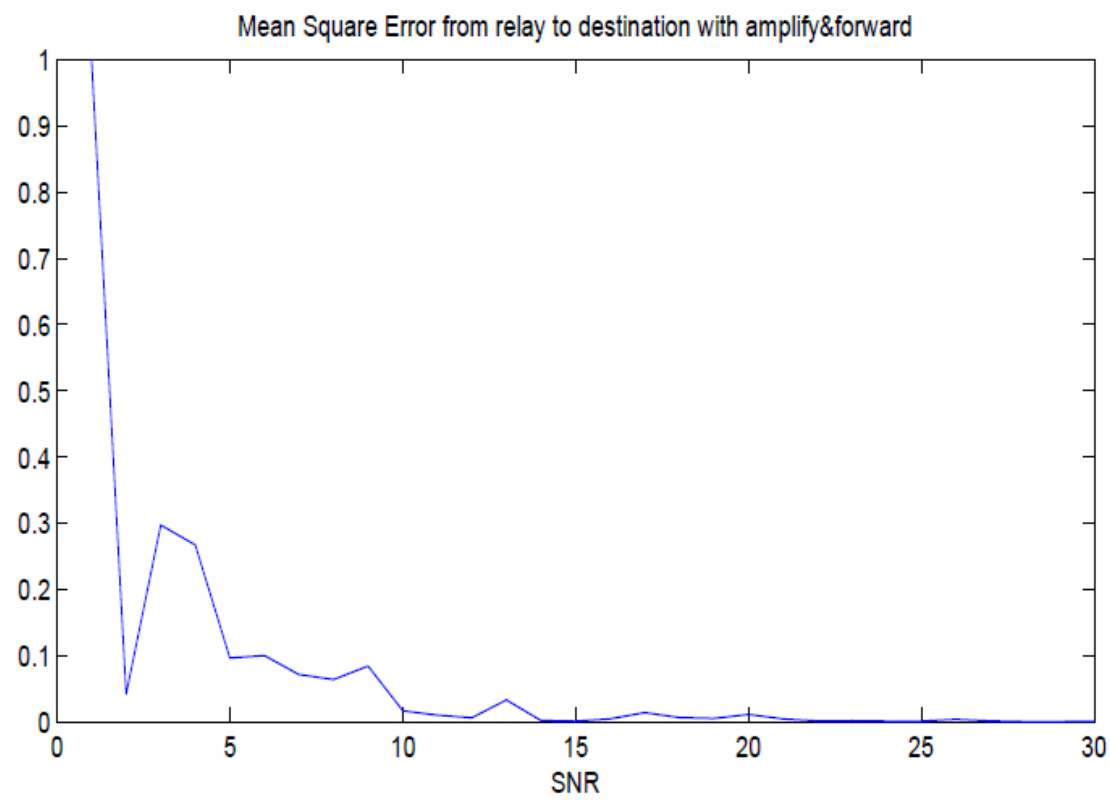

Fig. 7 Normalized mean square error of channel estimation

\section{References}

[1] D. Michalopoulos, H. Suraweera, G. Karagiannidis, and R. Schober, "Amplify and Forward Relay Selection with outdated Channel Estimates", IEEE Trans. Commun., vol. 60, no. 5, May 2012.

[2] Oyman O, Paulraj AJ., "Energy efficiency in MIMO relay networks under processing cost. In Conference on Information Science and Systems", Johns Hopkins University, March, 2005.

[3] W. C. Jakes, Microwave Mobile Communication, 2nd ed. Piscataway, NJ: IEEE Press, 1994.

[4] N. Kong and L. B. Milstein, "Average SNR of a generalized diversity Selection combining scheme," IEEE Commun. Lett., vol. 3, no. 3, pp.57-59, Mar. 1999.

[5] S. W. Kim, Y. G. Kim, and M. K. Simon, "Generalized selection combining based on the log-likelihood ratio," IEEE Trans. Commun.,vol. 52,no. 4,pp. 521-524, Apr. 2004.

[6] P. Gupta, N. Bansal, and R. K. Mallik, "Analysis of minimum selection GSC in Rayleigh fading," in Proc. IEEE Int. Conf. on Commun.(ICC'04), vol. 6, pp. 3364-3368, 2004.

[7] Suk Won Kim; Ha, D.S.; Reed, J.H., "Minimum selection GSC and adaptive low-power rake combining scheme," Circuits and Systems, 2003. ISCAS '03. Proceedings of the 2003 International Symposium on , vol.4, no., pp.IV-357,IV-360 vol.4, 25-28 May 2003.

[8] Hong-Chuan Yang, "Exact performance analysis of minimum-selection generalized selection combining (GSC)," IEEE International Conference on Communications,, vol.2, no., pp.1152,1156 Vol. 2, 16-20 May 2005.

[9] Hong-Chuan Yang, "New Results on Ordered Statistics And analysis of Minimum selection Generalized Selection Combining (GSC)", IEEE Trans. Commun., 2006.

[10] Yijia Fan1, Abdulkareem Adinoyi2, John S. Thompson1 and Halim Yanikomeroglu "Antenna combining for multi-antenna multi-relay channels" December 2006. 